\title{
Cyanuric Acid/Epichlorohydrin Energetic Prepolymers
}

\author{
Luís M. Pedroso*, Pedro Simões, António Portugal
}

Chemical Engineering Department, Faculty of Sciences and Technology, University of Coimbra, Pólo II, Pinhal de Marrocos, 3030-290 Coimbra (Portugal)

DOI: 10.1002/prep.200500023

\begin{abstract}
In this work new energetic prepolymers are synthesised and characterised. The structure of the prepolymers exhibits the 1,3,5$s$-triazine ring with lateral chains derived from the epichlorohydrin ring opening. The chlorine atoms in these precursors are here substituted by azido groups. The presence of these groups was confirmed by FTIR and ${ }^{1} \mathrm{H}$ NMR spectroscopy and elemental and thermal analysis. $\mathrm{OH}$ group content in the energetic prepolymers was found slightly lower than expected having in view the used inert precursors, while molar mass values were similar. Whereas the precursors show an endothermic thermal decomposition, the synthesised prepolymers show a clear exothermic thermal decomposition in DSC analyses. Due to the presence of the 1,3,5-striazine ring, the exothermic peaks were observed in a wide range of temperatures. The measured glass transition temperatures vary from $-15.5^{\circ} \mathrm{C}$ to $-43.3^{\circ} \mathrm{C}$. High densities and a wide range of viscosities were found.

The synthesised energetic prepolymers are interesting candidates for binder formulations for use in insensitive munitions (IM) due to the presence of the 1,3,5-s-triazine ring in the structure and their energetic character. The good curing properties found in a first test and the possibility to tailor the properties of the prepolymers are other attractive characteristics.
\end{abstract}

Keywords: Energetic Prepolymers, Cyanuric Acid, Epichlorohydrin, Azido Groups

\section{Introduction}

Prepolymers are used in energetic formulations like castcured explosives and propellants to give mechanical resistance, to increase the stability to impact, friction and heat, as well as to protect the energetic crystals. Energetic prepolymers also contribute to the released energy process. Due to its good characteristics [1], GAP is a widely used prepolymer that contains azido groups. It has an energy of decomposition of $685 \mathrm{~kJ} / \mathrm{mol}$, while nitrato groups show a much lower value of $167 \mathrm{~kJ} / \mathrm{mol}$ [2]. PAMMO and PBAMO are other prepolymers with azido groups [3].

Heterocyclic compounds have higher predicted thermal and shock stability and density than their analogue carboxylic compounds [4]. The higher nitrogen content also leads to lower oxygen requirements in energetic formulations [5]. The 1,3,5-s-triazine ring is present in RDX, a widely used

* Corresponding author; e-mail: miguel2@eq.uc.pt energetic material. Under conditions of high temperature and pressure it gives polycyclic systems, which are known to be burning rate modifiers [6]. DNAM increases the burning rate of PSAN/HTPB formulations, maintaining good shock stability properties [7]. MO5 is a heterocyclic energetic compound with azido groups and promising characteristics [8].

In this work energetic prepolymers containing the 1,3,5-striazine ring and azido groups in their structure were synthesised, from the reaction of inert precursors with sodium azide. Different prepolymers can be obtained by changing the epichlorohydrin:cyanuric acid ratio in the precursors. The hydroxyl end groups allow their cure with isocyanates.

\section{Experimental section}

\subsection{Materials and Equipment}

Reagents: cyanuric acid (>98\%) and epichlorohydrin (99\%) were obtained from Aldrich, tin (IV) chloride fuming and sodium azide (99\%) from Riedel-de Haën and N,Ndimethylformamide (99\%) from Fluka.

FTIR spectra were collected in a Nicolet 750 equipment, using a Golden Gate ATR accessory from Specac, where the products were analysed as prepared. Resolution was $4 \mathrm{~cm}^{-1}$ and the number of scans 64. NMR spectra were obtained in a Varian Unity 500 NMR spectrometer, operating at $499.824310 \mathrm{MHz}$ and $25^{\circ} \mathrm{C}$, using a $5 \mathrm{~mm}$ broad band probe. DMSO-d 6 (99.99\% D) from Riedel-de Haën was used as solvent.

Hydroxyl group content was measured by the acetic anhydride in pyridine method with 1 hour of reaction time at $98^{\circ} \mathrm{C}$ [9].

For the chromatographic studies the following equipment was used: Knauer degasifier, Knauer K-1001 pump, Polymer Laboratories PLgel $10 \mu \mathrm{m} 50 \mathrm{~nm}$ column in an Eldex CH150 oven at $70^{\circ} \mathrm{C}$ and a RI Knauer K-2301 detector. Osmometry was carried out in a Gonotec Osmomat 070. Temperature was set at $100^{\circ} \mathrm{C}$ and sampling time at 10 minutes. N,N-dimethylformamide $(99.9+\%$, HPLC grade from Aldrich) was used as solvent in both cases. 
Elemental analysis was performed in a Fisons Instruments EA 1108. DSC tests were done in a Polymer Laboratories DSC model PL DSC at $10.0 \mathrm{~K} \mathrm{~min}^{-1}$ and $\mathrm{N}_{2}$ atmosphere. DMTA experiments were performed in a Triton Tritec 2000 DMA. A steel plate in a closed $\mathrm{V}$ shape was used to hold the samples. Single cantilever $14 \mathrm{~mm}$ free sample length, displacement $50 \mu \mathrm{m}$, frequency $1 \mathrm{~Hz}$, heating rate $4.0 \mathrm{~K}$ $\mathrm{min}^{-1}$ and still air atmosphere were used in all experiments.

Viscosity was measured in a Haake Rheostress 1. Parallel plates geometry was used with lower plate diameter equal to $60.0 \mathrm{~mm}$ and upper plate diameter equal to $10 \pm 0.002 \mathrm{~mm}$, gap of $1.000 \mathrm{~mm}$ and temperatures of $30^{\circ} \mathrm{C}$ and $45^{\circ} \mathrm{C}$. Density was measured in an Accupyc 1330 helium pycnometer at $25^{\circ} \mathrm{C}$.

\subsection{Syntheses}

Inert precursors were used as starting materials in the syntheses of the energetic prepolymers. They were prepared by reacting cyanuric acid with epichlorohydrin $(\mathrm{ECH})$ in the presence of $\mathrm{SnCl}_{4}$ as catalyst and $\mathrm{N}, \mathrm{N}$-dimethylformamide (DMF) as solvent. ECH reacts with cyanuric acid and forms lateral chains bound to the nitrogen atoms of the ring [10]. This reaction differs from the traditional scheme where hydroxyl terminated initiators are used. The syntheses of the inert precursors were carried out in a $50 \mathrm{~mL}$ or $100 \mathrm{~mL}$ glass reactor equipped with a magnetic stirrer and reflux condenser in a temperature controlled oil heating bath. Predetermined quantities of cyanuric acid and DMF were added to the reactor. Stirring and heating were started and $\mathrm{ECH}$ was then added. The catalyst was gradually added for a period of 5 minutes. Table 1 shows the reagents and respective amounts used in the syntheses. All reactions were carried out at $70^{\circ} \mathrm{C}$ during $24 \mathrm{~h}$. Once reactions were finished, $50 \mathrm{~mL}$ of distilled water were added to the reaction products. After some minutes of stirring, the two phases were allowed to separate and the upper aqueous phase was removed. These steps were repeated twice and the obtained

Table 1. Reagents and respective amounts used in the syntheses of the precursors

\begin{tabular}{llccccc}
\hline & & PC14 & PC17 & PC13 & PC18 & PC19 \\
\hline Cyanuric acid & $\mathrm{mmol}$ & 199.86 & 117.11 & 91.34 & 91.40 & 54.54 \\
DMF & $\mathrm{mL}$ & 109.0 & 64.0 & 58.9 & 59.0 & 40.0 \\
ECH & $\mathrm{mmol}$ & 1000 & 1754 & 1883 & 1880 & 2199 \\
$\mathrm{SnCl}_{4}$ & $\mathrm{mmol}$ & 6 & 11 & 11 & 28 & 28 \\
\hline
\end{tabular}

products were dried overnight at $60^{\circ} \mathrm{C}$ in a ventilated oven. A final drying was carried out in a vacuum oven at $50^{\circ} \mathrm{C}$ and $1 \mathrm{~Pa}$.

The syntheses of the energetic prepolymers were carried out with the experimental setup used for the precursors. Predetermined quantities of the precursors were dissolved in the solvent DMF in the reactor. Sodium azide was then slowly added. The quantity of $\mathrm{NaN}_{3}$ used in the reactions, in a molar basis, was twice the mean value of chlorine present in the precursors, about $30 \%$ (mass) [10]. Table 2 presents the reagents and respective amounts used in the syntheses of the energetic prepolymers, which are named with an A after the designation of the precursor. All reactions were carried out at $75^{\circ} \mathrm{C}$ for 24 hours under continuous stirring. PC28A was synthesised with the same reagents and conditions of PC13A but at $100^{\circ} \mathrm{C}$. The ECH:cyanuric acid molar ratios of the precursors, based on elemental analysis results [10], are also indicated in Table 2. The reaction products were purified as the inert precursors, using $150 \mathrm{~mL}$ of distilled water.

\section{Results and Discussion}

\subsection{Spectroscopic Analysis}

Fig. 1 shows the FTIR spectra of PC13A and of its precursor PC13. The two spectra show several common features. The bands at 1455 and $850 \mathrm{~cm}^{-1}$ (not clearly visible) are due to the triazine ring and at $1685 \mathrm{~cm}^{-1}$ the stretching vibration of $\mathrm{C}=\mathrm{O}$ groups with endocyclic carbon is observed. These bands were less intense in the spectra of the prepolymers with higher ECH:cyanuric acid ratios. The band at about $3300 \mathrm{~cm}^{-1}$ is due to the $\mathrm{OH}$ stretching vibration. The bands between 2960 and $2870 \mathrm{~cm}^{-1}$ are due to the stretching vibrations of alkyl groups of the lateral chains. The band at $1090 \mathrm{~cm}^{-1}$ is attributed to $\mathrm{COH}$ and the band at $1060 \mathrm{~cm}^{-1}$ to COC stretching vibrations [11].

The major differences are the bands at 2094 and $2025 \mathrm{~cm}^{-1}$ in the PC13A spectrum, due to the azido groups. The effect of the replacement of chlorine atoms is also observed by the different heights of the bands at 760 and $698 \mathrm{~cm}^{-1}$, attributed to chloroalkyl groups [11]. PC13A shows a weak band at $760 \mathrm{~cm}^{-1}$, attributed to ring vibrations, while the band at $698 \mathrm{~cm}^{-1}$ is not observed. The intense band at $1282 \mathrm{~cm}^{-1}$ in PC13A spectrum is due to $\mathrm{CH}_{2} \mathrm{~N}_{3}$ groups $[1,12]$. No other relevant differences are observed.

The band due to azido groups at $2100 \mathrm{~cm}^{-1}$ was more intense in the spectra of the prepolymers synthesised from

Table 2. Reagents and respective amounts used in the syntheses of the prepolymers

\begin{tabular}{lllllll}
\hline & & PC14A & PC17A & PC13A & PC18A & PC19A \\
\hline Inert precursor & $\mathrm{g}$ & 50.6388 & 50.1215 & 50.0105 & 25.3644 & 25.3445 \\
DMF & $\mathrm{mL}$ & 135.0 & 135.0 & 135.0 & 68.0 & 68.0 \\
NaN3 & $\mathrm{g}$ & 70.0761 & 70.0020 & 70.0416 & 34.4330 & 34.8297 \\
ECH:cyanuric acid ratio of inert precursor & $\mathrm{mol} / \mathrm{mol}$ & $3.4: 1$ & $5.2: 1$ & $7.5: 1$ & $10.2: 1$ & $14.5: 1$ \\
\hline
\end{tabular}




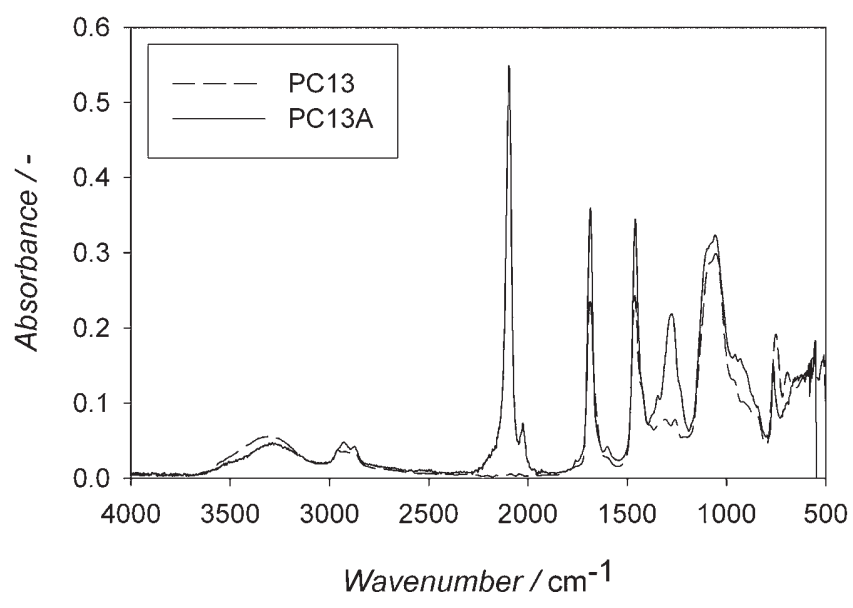

Figure 1. IR spectra of PC13A and its inert precursor PC13.

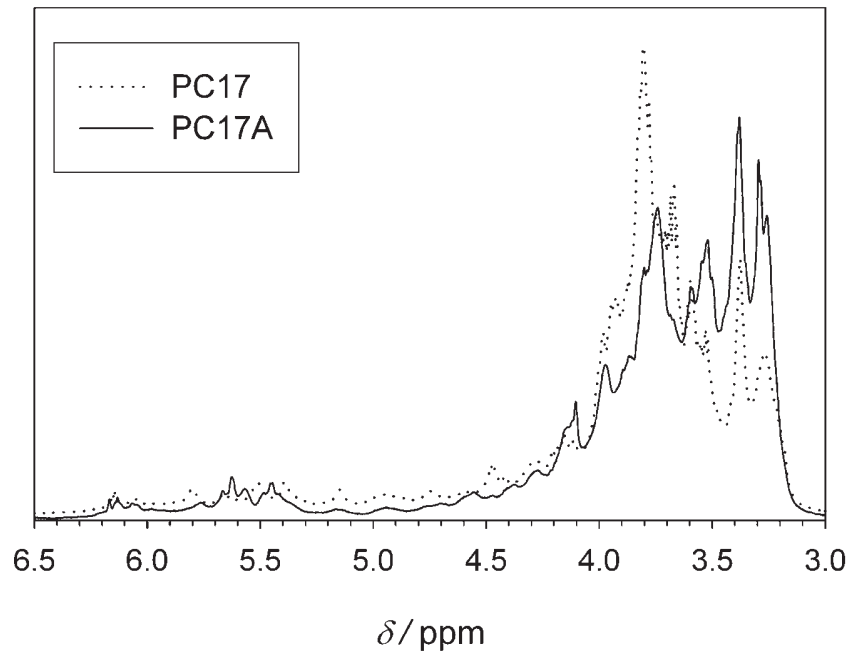

Figure 2. ${ }^{1} \mathrm{H}$ NMR spectra of D6-DMSO solutions of PC17A and its inert precursor PC17 at $499.824310 \mathrm{MHz}$ and $25^{\circ} \mathrm{C}$.

precursors with higher ECH:cyanuric acid ratios. This was expected since chlorine is introduced by $\mathrm{ECH}$ and its higher content leads to a higher substitution.

Fig. 2 shows the ${ }^{1} \mathrm{H}$ NMR spectra of PC17A and its inert precursor PC17 between $3.0 \mathrm{ppm}$ and $6.5 \mathrm{ppm}$. Outside this range, only low intensity peaks from the residual protons of DMSO- $\mathrm{d}_{6}$ and the protons of DMF were observed. The peaks between $4.6 \mathrm{ppm}$ and $5.9 \mathrm{ppm}$ are assigned to protons of hydroxyl groups [10]. In the range $3.2-4.5 \mathrm{ppm}$ the signals of the protons in the lateral chains are observed [10]. The peaks in the spectrum of PC17 at 3.6-3.8 ppm, due to protons from $\mathrm{CH}_{2} \mathrm{Cl}$ groups, are weak in the spectrum of PC17A, which shows instead intense signals at 3.3-3.5 ppm due to the protons of $\mathrm{CH}_{2} \mathrm{~N}_{3}$ groups [1]. The protons of the $\mathrm{CH}_{2}$ groups bound to the triazine ring show signals at 3.7$4.0 \mathrm{ppm}$ and the protons from $\mathrm{CH}_{2}$ or $\mathrm{CH}$ groups bound to ether bonds or hydroxyl groups show signals at $3.5 \mathrm{ppm}$ [10]. The proposed structures of the energetic prepolymers are presented in Fig. 3, where the lateral chains were formed by one (Fig. 3a) or more (Fig. 3b) ECH molecules.<smiles>[R]n1c(=O)n([R])c(=O)n(CC(O)CN)c1=O</smiles><smiles>[R]OC(CN)COC(CN)CN(C)O</smiles>

b)

Figure 3. Proposed structures for the lateral chains of PC17A. $\mathrm{R}_{1}$ and $\mathrm{R}_{2}$ are lateral chains. The $\mathrm{N}$ atom in $\mathrm{b}$ ) is from the triazine ring. $R_{3}$ is a proton or the continuation of the lateral chain.

Table 3. Hydroxyl groups content of the prepolymers (mass- $\%$ )

\begin{tabular}{lll}
\hline Prepolymer & OH $(\%)$ & Standard Deviation \\
\hline PC13A & 5.84 & 0.44 \\
PC18A & 4.55 & 0.05 \\
PC19A & 3.52 & 0.10 \\
\hline
\end{tabular}

\subsection{Determination of Hydroxyl Group Content}

Table 3 shows the results for hydroxyl group content in the prepolymers. Two analyses have been performed for each prepolymer. As observed in the precursors [10], hydroxyl group content decreases as the ECH:cyanuric acid ratio increases. When compared to $\mathrm{OH}$ content in the inert precursors, the energetic prepolymers show a decrease between $2.5 \%$ for PC13A and $1.0 \%$ for PC19A (absolute values).

\subsection{Molar mass}

Table 4 shows the measured molar masses of the prepolymers by chromatography. The precursors show broad molar mass distributions [10], being this also observed in the energetic prepolymers by the differences in $M_{w}, M_{n}$ and $M_{p}$ (mass of the peak). The relatively low masses are due to the solvent used in the syntheses of the precursors [10]. The energetic prepolymers have molar mass distributions similar to the precursors, showing that the substitution of chlorine by azido groups does not affect this parameter significantly. The $M_{n}$ value of PC19A measured by osmometry is $495 \mathrm{~g} \mathrm{~mol}^{-1}$. This shows that the measuring technique influences the results, and the slight widening of the 
Table 4. Molar mass values of the prepolymers

\begin{tabular}{llll}
\hline Prepolymer & $\begin{array}{l}M_{w} \\
\mathrm{~g} \mathrm{~mol}^{-1}\end{array}$ & $\begin{array}{l}M_{n} \\
\mathrm{~g} \mathrm{~mol}^{-1}\end{array}$ & $\begin{array}{l}M_{p} \\
\mathrm{~g} \mathrm{~mol}^{-1}\end{array}$ \\
\hline PC14A & 465 & 269 & 594 \\
PC17A & 411 & 212 & 559 \\
PC13A & 419 & 257 & 494 \\
PC18A & 354 & 181 & 600 \\
PC19A & 382 & 228 & 640 \\
\hline
\end{tabular}

Table 5. Elemental analysis results

\begin{tabular}{lllllr}
\hline & $\begin{array}{l}\mathrm{C} \\
\%\end{array}$ & $\begin{array}{l}\mathrm{N} \\
\%\end{array}$ & $\begin{array}{l}\mathrm{H} \\
\%\end{array}$ & $\begin{array}{l}\text { O } \\
\%\end{array}$ & \multicolumn{1}{c}{$\begin{array}{l}\text { Other } \\
\%\end{array}$} \\
\hline PC14A & 36.677 & 29.254 & 6.898 & 21.798 & 5.375 \\
PC17A & 35.935 & 25.404 & 6.093 & 20.124 & 12.445 \\
PC13A & 35.184 & 27.926 & 4.799 & 19.377 & 12.716 \\
PC18A & 35.473 & 26.224 & 6.587 & 20.305 & 11.412 \\
PC19A & 36.054 & 27.374 & 5.548 & 18.432 & 12.593 \\
PC28A & 33.736 & 29.617 & 5.306 & 22.208 & 9.133 \\
\hline
\end{tabular}

chromatographic peaks due to the column leads to lower $M_{n}$ values.

\subsection{Elemental Analysis}

Table 5 shows the elemental analysis results for the energetic prepolymers.

The $\mathrm{N}$ content in the precursors varies from $2.9 \%$ (PC19) to $9.6 \%$ (PC14) [10]. The values found for the energetic prepolymers are significantly higher, due to the introduction of the $-\mathrm{N}_{3}$ groups. However, the values in the column "Other" (see Table 5) suggest that some chlorine atoms were not substituted. PC28A $\left(100^{\circ} \mathrm{C}\right)$ shows a higher $\mathrm{N}$ content and a lower value in the column "Other" than PC13A. This indicates that more severe reaction conditions are required for complete substitution. Sodium and tin contents were also measured, giving negligible results. The $\mathrm{N}$ content is higher for the prepolymers synthesised with precursors with low and high ECH:cyanuric acid ratios. In the first case, the nitrogen content from cyanuric acid in the prepolymers is high, while in the latter the higher chlorine content leads to a higher substitution by azido groups. Carbon content is similar for all compounds and is slightly lower than the precursors [10] due to the higher mass of the azido group when compared to chlorine atom. The same can be said for oxygen.

\subsection{Thermal Analysis}

Fig. 4 shows the DSC analyses of PC13A and PC13. Similar masses were used in both analyses. PC13 shows an endothermic decomposition involving low energy. The behaviour of PC13A is drastically different, with a strong exothermic peak due to the scission of azido groups. The onset temperature of the decomposition is similar in both

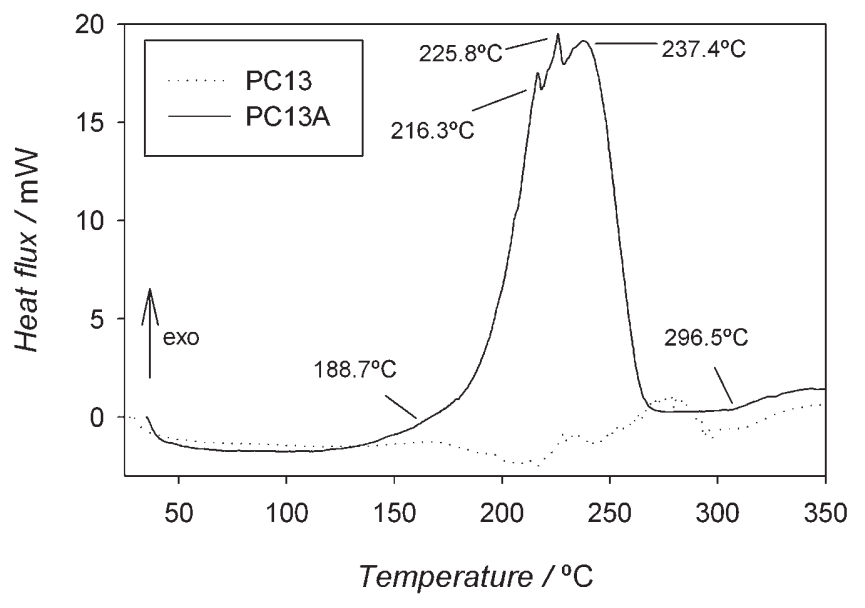

Figure 4. DSC of PC13A and its precursor PC13.

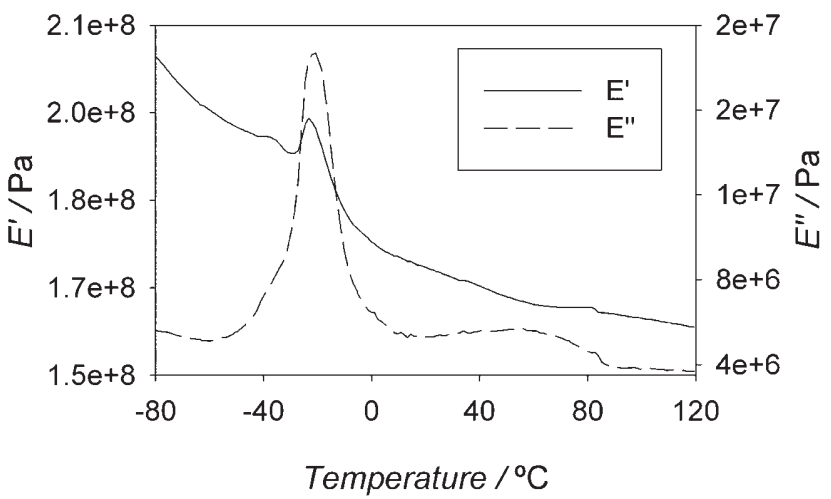

Figure 5. DMTA analysis of PC13A.

cases. The exothermic thermal decomposition of PC13A is observed in a wide temperature range. Small peaks are observed within the main peak. This behaviour can be attributed to the exothermic decomposition and the high thermal resistance of the triazine ring [13], with opposite effects.

The onset temperature of the decomposition of the energetic prepolymers increased with the ECH:cyanuric acid ratio, from $169.0^{\circ} \mathrm{C}$ for PC14A to $201.2^{\circ} \mathrm{C}$ for PC19A, while the exothermic peak became narrower. This confirmed the effect of the cyanuric acid part of the molecules in the decomposition. The effect of the initiators in the thermal decomposition temperature is also observed in GAP prepolymers [1].

Fig. 5 shows the DMTA analysis of PC13A. The values of $E^{\prime}$ and $E^{\prime \prime}$ are only indicative due to the used steel support. Since it has no influence in the variation of $E^{\prime}$ and $E^{\prime \prime}$, the variations in the curves are due to the sample alone.

The peak in $E^{\prime \prime}$ curve is due to the glass transition temperature $\left(T_{g}\right)$ of PC13A. A noticeable effect of the relaxation of the chains at this temperature is observed by the peak in $E^{\prime}$ curve [14]. A second wide and much less intense peak in $E^{\prime \prime}$ is observed between $15^{\circ} \mathrm{C}$ and $85^{\circ} \mathrm{C}$, with 
Table 6. Glass transition temperatures of the prepolymers

\begin{tabular}{llllll}
\hline Prepolymer & & PC17A & PC13A & PC18A & PC19A \\
\hline$T_{g}$ & ${ }^{0} \mathrm{C}$ & -15.5 & -10.8 & -28.2 & -43.8 \\
\hline
\end{tabular}

Table 7. Densities of PC13A, PC18A and PC19A at $25^{\circ} \mathrm{C}$

\begin{tabular}{ll}
\hline Prepolymer & $\begin{array}{l}\text { Density }{ }^{(a)} \\
\mathrm{g} \mathrm{cm}^{-3}\end{array}$ \\
\hline PC13A & $1.4038 \pm 0.0004$ \\
PC18A & $1.4021 \pm 0.0013$ \\
PC19A & $1.3591 \pm 0.0010$ \\
\hline
\end{tabular}

(a) The uncertainties are referred to a confidence level of $95 \%$.

maximum value at around $55^{\circ} \mathrm{C}$. At this temperature a small onset in $E^{\prime}$ curve is observed. This second transition was more intense for PC14A at $25.0^{\circ} \mathrm{C}$ and less intense for PC18A at $55^{\circ} \mathrm{C}$. PC19A showed this peak at $-10^{\circ} \mathrm{C}$ in $E^{\prime \prime}$ curve and for PC17A it was not observed. This second event is attributed to a transition involving the triazine ring part of the molecules. Table 6 shows the glass transition temperatures of the prepolymers, determined by $E^{\prime \prime}$ peak temperature. PC14A is not listed due to uncertainties in the attribution. The $T_{g}$ of the prepolymers decreases as the $\mathrm{ECH}$ :cyanuric acid ratio increases, showing the importance of the lateral chains for this parameter.

The cure of PC19A was performed with isophorone diisocyanate and a rubber like material was obtained. The formation of some gas bubbles in the sample, possibly due to entrapped air or residual moisture, hindered a more extensive test program of the cured compound. However, this result showed that the prepolymers can be cured with current isocyanates to yield energetic polyurethane elastomers.

\subsection{Viscosity and Density}

The viscosity of PC14A was too high to be measured by the equipment used. PC17A also showed a high viscosity, $2400 \mathrm{~Pa} \mathrm{~s}$ at $45^{\circ} \mathrm{C}$ and $1 \mathrm{~s}^{-1}$. The viscosities of PC13A, PC18A and PC19A measured at $30^{\circ} \mathrm{C}$ and $10 \mathrm{~s}^{-1}$ were 288 , 670, and $23 \mathrm{~Pa}$ s, respectively. This shows that ECH:cyanuric acid ratio can be adjusted to get a wide range of viscosities. This feature can be tailored for the desired formulation.

Table 7 shows the measured densities of the prepolymers. The densities of PC13A and PC18A are similar, while the density of PC19A is lower due to the higher ECH:cyanuric acid ratio. The values found are higher than those of GAP of $1.29 \mathrm{~g} \mathrm{~cm}^{-3}$ [15] due to the presence of the triazine ring.

\section{Conclusions}

In this work energetic prepolymers were synthesised starting from precursors with the 1,3,5-s-triazine ring and lateral chains derived from ECH ring opening. The chlorine atoms were substituted by azido groups by reaction with sodium azide. The presence of these groups was confirmed by IR and ${ }^{1} \mathrm{H}$ NMR spectroscopy and elemental and thermal analysis. DSC analysis showed a clear exothermic peak for all prepolymers, confirming the exothermic thermal decomposition. The peaks were observed in a wide temperature range, indicating a gradual energy release. The choice of the $\mathrm{ECH}$ :cyanuric acid ratio in the precursors leads to different final properties of the energetic prepolymers. This permits to tailor the synthesised prepolymers to meet different needs in energetic formulations, making them attractive ingredients for energetic binders.

\section{References}

[1] Y. Mohan, M. Raju and K. Raju, Synthesis, Spectral and DSC Analysis of Glycidyl Azide Polymers Containing Different Initiating Diol Units, J. Appl. Polym. Sci. 2004, 93, 2157.

[2] E. Diaz, P. Brousseau, G. Ampleman and R. Prud'homme, Heats of Combustion and Formation of New Energetic Thermoplastic Elastomers Based on GAP, PolyNIMMO and PolyGLYN, Propellants, Explos., Pyrotech. 2003, 28 (3), 101.

[3] C. J. Campbell, Energetic Oxetane Propellants, U.S. Patent 6217682, 2001, Cordant Technologies Inc., USA.

[4] R. Chapman, W. Wilson, J. Fronabarger, L. Merwin and G. Ostrom, Prospects of Fused Polycyclic Nitroazines as Thermally Insensitive Energetic Materials, Thermochim. Acta 2002, 384, 229

[5] J. Oxley, J. Smith, and H. Chen, Thermal Decomposition of High-Nitrogen Energetic Compounds-Dihydrazido-S-Tetrazine Salts, Thermochim. Acta 2002, 384, 91.

[6] T. B. Brill, Connecting the Chemical Composition of a Material to its Combustion Characteristics, Prog. Energy Combust. Sci. 1992, 18, 91.

[7] P. Simões, L. Pedroso, and A. Portugal, New Propellant Component, Part II. Study of a PSAN/DNAM/HTPB Based Formulation, Propellants, Explos., Pyrotech. 2001, 26 (6), 278.

[8] A. Kulikov, I. Ovchinnikov, S. Molotov and N. Makhova, Synthesis of Furoxan Derivatives Based on 4-Aminofuroxan3-Carboxylic Acid Azide, Russ. Chem. Bull. Int. Ed. 2003, 52 (8), 1822.

[9] C. L. Ogg, W. L. Porter, and C. O. Willits, Determining the Hydroxyl Content of Certain Organic Compounds; Macro and Semimacro Methods, Ind. Eng. Chem., Anal. Ed. 1945, 17, 394.

[10] L. Pedroso, P. Simões and A. Portugal, Cyanuric Acid/ Epichlorohydrin Prepolymers, J. Appl. Polym. Sci. 2005 (in press)

[11] N. P. G. Roeges, A Guide to Complete Interpretation of Infrared Spectra of Organic Structures, John Wiley \& Sons, Chichester 1994.

[12] M. Eroglu, O. Guven, Spectroscopic and Thermal Characterization of Poly(glycidyl Azide) Converted from Polyepichlorohydrin, J. Appl. Polym. Sci. 1996, 60, 1361.

[13] J. M. E. Quirke, The Structure, Reactions, Synthesis and Uses of Heterocyclic Compounds. Vol. 3. in J. Boulton, A. McKillop (Eds) Comprehensive Heterocyclic Chemistry, Pergamon Press, Oxford 1984.

[14] K. Menard, Dynamic Mechanical Analysis - A Pratical Introduction, CRC Press, Boca Raton 1999.

[15] A. Provatas, Energetic Polymers and Plasticizers for Explosive Formulations - A Review of Recent Advances, DSTO - Tr 0966, 2000, Department of Defense - Defense Science \& Technology Organisation, Melbourne, Australia. 


\section{Symbols and Abbreviations}

DNAM 4,6-Dinitramino-1,3,5-triazine-2(1H)-one

$\mathrm{ECH} \quad$ Epichlorohydrin

GAP Glycidyl azide polymer

HTPB Hydroxyl-terminated polybutadiene

MO5

PAMMO

PBAMO

PSAN

RDX
4-Aminofuroxan-3-carboxylic acid azid

Poly(3-azidomethyl-3-methyloxetane))

Poly(3,3-bis(azidomethyl)oxetane)

Phase stabilized ammonium nitrate

Cyclo-1,3,5-trimethylene-2,4,6-trinitramine

\section{Acknowledgements}

We are grateful to Dr. M. Margarida C. A. Castro from the Biochemistry Department - NMR Center and Center for Neuroscience and Cell Biology, University of Coimbra, for her assistance in the NMR studies.

(Received December 13, 2004, revised version May 10, 2005; Ms 2004/048)

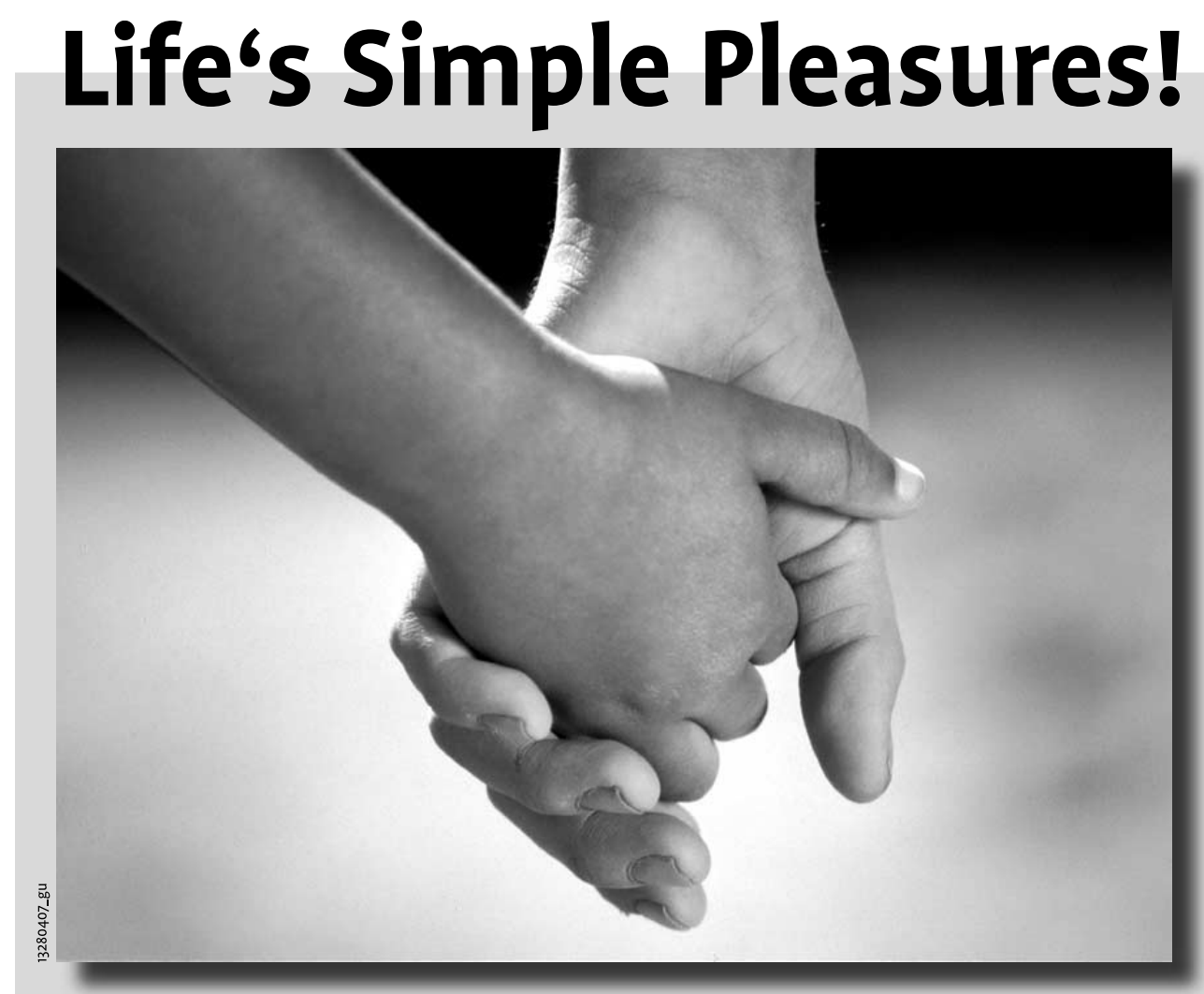

No need to waste precious time looking for the right information - Register now for the free Wiley-VCH Alerting Service.

It's simple and it's fast.

To receive regular news per e-mail tailored precisely to your needs and interests, just fill in the registration form at www.wiley-vch.de/home/pas/ 